\title{
The use of GV-971 induces liver injury in an Alzheimer's disease patient
}

\author{
Hao-Ran Cheng ${ }^{1}$, Cai-Yun Wen ${ }^{1}$, Chunhong Zhang², William Kwapong ${ }^{3}$, and zhen wang ${ }^{2}$ \\ ${ }^{1}$ Wenzhou Medical University First Affiliated Hospital \\ ${ }^{2}$ Affiliation not available \\ ${ }^{3}$ West China Hospital of Medicine
}

July 6,2020

\begin{abstract}
A 57-year old woman with Alzheimer's disease developed liver injury 4 days after starting GV-971 therapy. This woman had no history of drug allergy before her AD diagnosis. After started her GV-971 medication (150 mg bid) while taking donepezil and memantine hydrochloride, she presented with jaundice of the skin and sclera, loss of appetite and fatigue. Laboratory examination suggested that liver function was abnormal with serum levels of alanine transaminase (ALT) (737 U/L), aspartate transaminase (1006 U/L). And Magnetic Resonance Imaging (MRI) showed the interstitial edema of the portal vein and the thickened gallbladder wall, which suggested the possibility of active liver disease or liver injury. Our report showed that GV-971 may lead to liver injury and we suggest that clinicians should monitor liver functions when using GV-971 in AD patients.
\end{abstract}

\section{Introduction}

Sodium oligomannate capsules (GV-971; Green Valley Pharmaceuticals, Shanghai, China), a regulator of gut microbiota derived from marine algae, was approved by the China Food and Drugs Administration in November 2019 for the treatment of mild to moderate patients diagnosed with Alzheimer's diseases, to improve cognitive function ${ }^{1}$. Previous AD treatment was focused on restoring the balance of neurotransmitters in the brain; however, increasing evidence has suggested the association between gut dysbiosis and AD progression $^{1}$. The drug, GV-971, purportedly works by modifying gut bacteria, which has been proven to reduce brain inflammation in genetically engineered mice afflicted with $\mathrm{AD}$. The role of gut microbiota in AD patients remains obscure. In this report, we shed light on the effect of GV-971 in an AD patient.

\section{Results - Case presentation}

A 57-year old woman with Alzheimer's disease developed liver injury 4 days after starting GV-971 therapy. This woman had no history of drug allergy before her AD diagnosis. She had been diagnosed with Alzheimer's disease for 10 years and has been on donepezil and memantine hydrochloride medication. Her recent followup in October 2019, showednormal liver function test but serum $\gamma$-glutamyltransferase (79 U/L) was slightly elevated; her Mini-Mental State Examination (MMSE) score was 0 and her Montreal Cognitive Assessment (MoCA) score was 1.

On January 24, 2020, this patient started her GV-971 medication (150 mg bid) while taking donepezil and memantine hydrochloride; 4 days after her GV-971 therapy, she presented to the hospital with jaundice of the skin and sclera, loss of appetite and fatigue. On January 30, 2020, she stopped taking GV-971 after consultation. During drug withdrawal, jaundice started to fade and appetite returned to normal. Due to the coronavirus outbreak, the patient was hospitalized on February 18, 2020, and started liver protection therapy. 
Serum levels of total bilirubin $(411 \mu \mathrm{mol} / \mathrm{L})$, direct bilirubin $(337 \mu \mathrm{mol} / \mathrm{L})$, alanine transaminase (ALT) $(737 \mathrm{U} / \mathrm{L})$, aspartate transaminase $(1006 \mathrm{U} / \mathrm{L})$, alkaline phosphatase $(165 \mathrm{U} / \mathrm{L}), \gamma$-glutamyltransferase $(144$ $\mathrm{U} / \mathrm{L})$, eosinophil count $\left(0.02 \times 10^{9} / \mathrm{L}\right)$. Magnetic Resonance Imaging (MRI) showed the interstitial edema of the portal vein and the thickened gallbladder wall, which showed the possibility of active liver disease or liver injury as shown in Figure 1. Analysis of serum protein electrophoresis excluded the diagnosis of autoimmune hepatitis and Wilson's disease. A liver biopsy was not done because the patient could not cooperate.

During hospitalization, the patient's symptoms and liver function gradually improved.

\section{Discussion}

Drug-induced liver injury (DILI) is a liver injury induced by all types of prescription or non-prescription drugs; it is a common drug reaction and one of the leading causes of drug withdrawal which results in liver failure and death ${ }^{2}$. A recent review suggested that the contributing factor of DILI may be drug interactions and multisystem involvement ${ }^{3}$. Nonetheless, it's a challengeto diagnose DILI due to insufficient objective and specific diagnostic indices and DILI has no particular treatment methods ${ }^{4,5}$.

Our present case report showed that our patient presented with jaundice, loss of appetite and fatigue and her radiologic examination showed active liver injury. However, symptoms of liver injury and higher levels of bilirubin and liver enzymes improved after stopping the intake of GV-971. The clinical course of her disease and clinical examinations suggested that GV-971 was the most likely cause of DILI in our patient. RousselUclaf Causality Assessment Method (RUCAM), a scale used in assessing causality of drug-induced liver injury, was used in our current case to determine the cause (GV-971) in our current report.

According to the EASL Clinical Practice Guidelines, DILI can be classified as direct DILI and idiosyncratic DILI (IDILI); IDILI can be further divided into immunologically IDILI and genetically IDILI according to the patient's immune response. Additionally, immunologically IDILI can manifest itself as hypersensitivity and autoimmunity. The liver injury that occurred four days after starting GV-971 therapy and the normal reports of serum immunology and liver antigens spectrum showed hypersensitivity which led us to suggest our patient had immunological IDILI. Based on the revised DILI criteria by the Council for International Organizations of Medical Sciences (CIOMS), our patient showed the hepatocellular pattern of IDILI ${ }^{6,7}$. However, our patient did not present with systemic allergies such as fever, rash or eosinophilia; this may be due to the patient's immune reaction to the drug or the duration of drug usage.

\section{Conclusion}

Our current report is the first to show that GV-971 is associated with drug-induced hepatitis in an AD patient. Our report showed that GV-971 may lead to liver injury and we suggest that clinicians should monitor liver functions when using GV-971 in AD patients.

\section{Data availability statement}

The data that support the findings of this study are avaliable on request from the corresponding author. Research data are not shared due to privacy or ethical restrictions.

\section{Disclosure}

The authors declare no competing interests.

\section{Contribution statement}

Hao-Ran Cheng: Conceptualization, Data Curation, Formal analysis and Writing original draft. Cai-Yun Wen: Data Curation and Formal analysis. Chun-Hong Zhang: Data Curation and Formal analysis. William Robert Kwapong: Conceptualization and Writing-review \& editing. Zhen Wang: Funding acquisition, Resources and Writing-review \& editing.

\section{Funding}


This work was supported by the Projects of Provincial Natural Science Foundation of Zhejiang (No. LY19H090013) and the Projects of Medical Health Science and Technology of Zhejiang (No. 2020KY637).

\section{References}

1. Wang X, Sun G, Feng T, et al. Sodium oligomannate therapeutically remodels gut microbiota and suppresses gut bacterial amino acids-shaped neuroinflammation to inhibit Alzheimer's disease progression. Cell research 2019;29:787-803.

2. Devarbhavi H, Choudhury AK, Sharma MK, et al. Drug-Induced Acute-on-Chronic Liver Failure in Asian Patients. Am J Gastroenterol 2019;114:929-937.

3. Asrani SK, Devarbhavi H, Eaton J, Kamath PS. Burden of liver diseases in the world. J Hepatol 2019;70:151-171.

4. Liakoni E, Ratz Bravo AE, Terracciano L, Heim M, Krahenbuhl S. Symptomatic hepatocellular liver injury with hyperbilirubinemia in two patients treated with rivaroxaban. JAMA Intern Med 2014;174:1683-1686.

5. European Association for the Study of the Liver. Electronic address eee, Clinical Practice Guideline Panel C, Panel m, representative EGB. EASL Clinical Practice Guidelines: Drug-induced liver injury. J Hepatol 2019;70:1222-1261.

6. Mullins C, Beaulac K, Sylvia L. Drug-Induced Liver Injury (DILI) With Micafungin: The Importance of Causality Assessment. Ann Pharmacother 2019:1060028019892587.

7. Andrade RJ, Chalasani N, Bjornsson ES, et al. Drug-induced liver injury. Nature reviews Disease primers 2019;5:58.

\section{Figure legend}

Figure 1. Magnetic Resonance Imaging (MRI) of the patient. MRI showed the wall of the gallbladder thickened and had line shadows next to the portal vein branches which were low signal in T1WI and high signal in T2WI. This showed the interstitial edema of the portal vein branches and the thickened gallbladder wall, which revealed the possibility of active liver disease or liver injury. 


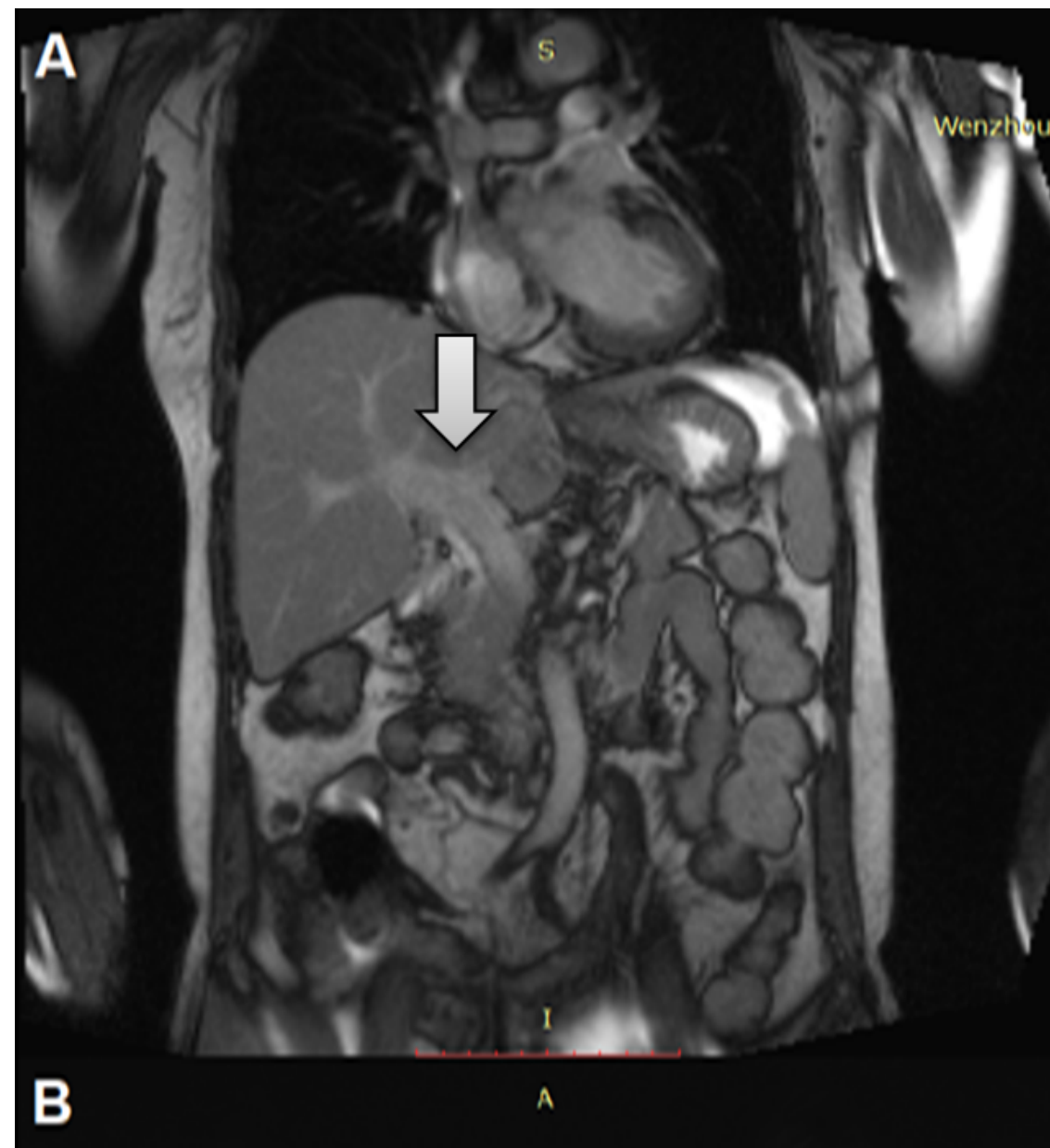

Wenzhou
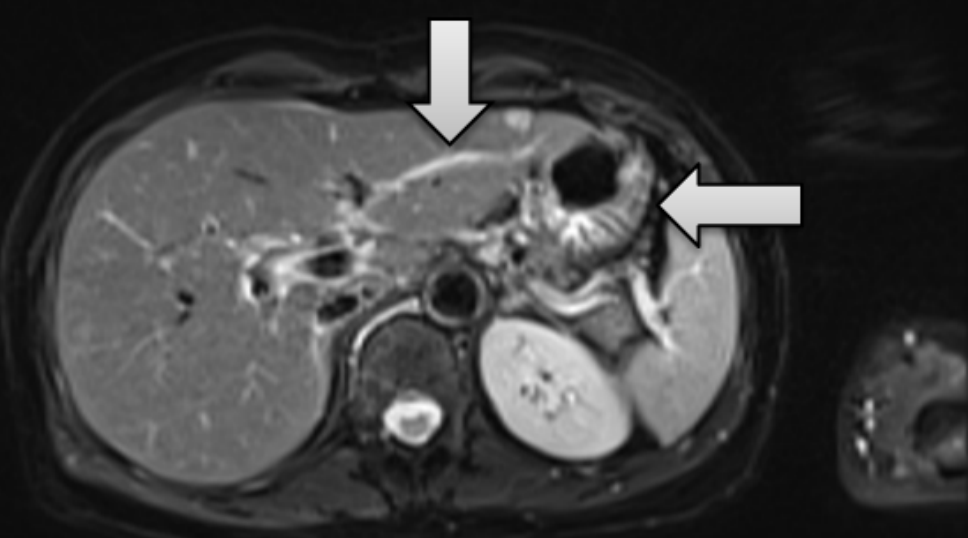

C 